\title{
Geo-Epidemiology of Tobacco Smoking in Europe and the impact of Twelve Tobacco Control Measures
}

\author{
Pistolla Georgia, Sifaki-Pistolla Dimitra \\ Faculty of Medicine, University of Crete, Iraklion, Greece
}

Email address:

geopist@gmail.com (G. Pistolla), spdimi11@gmail.com (D. Sifaki-Pistolla)

\section{To cite this article:}

Pistolla Georgia, Sifaki-Pistolla Dimitra. Geo-Epidemiology of Tobacco Smoking in Europe and the impact of Twelve Tobacco Control Measures. Science Journal of Public Health. Special Issue: Spatial Analysis and Mathematics in Health Research, during Times of Global Socio-Economic Instability. Vol. 3, No. 3-1, 2015, pp. 24-29. doi: 10.11648/j.sjph.s.2015030301.15

\begin{abstract}
Background: Tobacco use is one of the major causes of premature death worldwide. The purpose of the present study is to examine which of the known evidence-based control measures actually work, and indentify their preventive or risky effects, in order to determine the various risk areas for any daily tobacco use in Europe. This is a cross-sectional geoepidemiological study using secondary database data from the World Health Organization. Methods: Data from 2009 were used for all European countries. Daily smoking of any tobacco product (DSTP) and 12 other factors referring to tobacco control, comprise the main under study parameters. The final sample consisted of subjects from 45 European countries. First, the data management was fulfilled. Then, spatial statistics were performed in Arcmap 10 (GIS). Moran's I and OLS spatial regression model were applied for the detection of spatial correlations and the high risk areas. Results: High spatial heterogeneity was demonstrated through the DSTP distribution. Greece and Austria have the highest rates (38-49\%), Bulgaria, Albania, Bosnia Herzegovina, and Russia follow with 30-37\%. Three out of the twelve factors examined were found to be random. The rest of them were used in the regression model, highlighting the high risk areas. Greece presented the highest risk $(\operatorname{Exp} B=2.9 ; 95 \% \mathrm{CI}=2.183-3.326)$, Bulgaria, Turkey, Serbia and other countries followed $($ Exp $\mathrm{B}=2.7 ; 95 \% \mathrm{CI}=1.937$ 3.013), whereas Finland, Norway and UK proved to be of no present risk (Exp B=0.76; 95\% CI= 0.258-0.936). Conclusions: Geo-epidemiology of tobacco usage monitors the phenomenon's dynamics, effectively. It offers an insight in applying measures, co-examining for the first time, the effects of twelve measures of tobacco control as possible preventive or risk factors.
\end{abstract}

Keywords: Tobacco Use, Control Measures, Spatial Analysis

\section{Introduction}

Tobacco use is one of the major causes of premature death worldwide [1]. The World Health Organization (WHO) estimates that tobacco use in the 20th century resulted in 100 million deaths worldwide, when at the same time more than one billion deaths are already attributed to tobacco use in the $21^{\text {st }}$ century [2,3]. Approximately 4000 chemicals have been detected in tobacco smoke, 60 of which are known or suspected to be carcinogens [4]. Hence, various diseases such as lung cancer, cardiovascular diseases or diseases of the respiratory system are caused by or deteriorated with tobacco use $[4,5]$.

Tobacco use should be reduced through comprehensive control measures and policies. Currently, such policies include various actions at an international level, like smoke- free public places, smoking cessation services, tobacco taxes and mass media campaigns [6]. For instance, it is estimated that $40 \%$ of the Greek adults are smokers, who most of the times smoke even in public places, despite the relevant legislation enforcement [7]. Such legislation in Greece refers to implementation of smoking ban in public and private health care services, educational institutions, public transportations and public services areas [8]. Similar antitobacco laws have been recently implemented by the Italian Government. Italy has enriched its tobacco control laws by forbidding smoking in public places, increasing taxes and prices of cigarettes and applying strict anti-tobacco advertisement and media law [9]. These measures helped in reducing the tobacco use in that country.

According to Thomas et al. and Kenji Shibuya et al., there are various tobacco control measures which affect its use and these include the following: price control and tax measures, 
protection from exposure to environmental tobacco smoke, regulation around the disclosure of the contents of tobacco products, informative packaging and labeling, education, communication of risks, training, public awareness, and comprehensive ban and restriction on tobacco advertising, promotion, and sponsorship, tobacco dependence and cessation measures $[10,11]$. These are measures that target to tobacco control and could be characterized as preventive measures. But what happens when (and if) these measures turn into risk factors?

Tobacco use of any tobacco product has already been studied extensively, yet there are few studies which test its spatial parameter through spatial statistics $[12,14]$. The purpose of the present study is to examine which of the known evidence-based control measures actually work, and indentify their preventive or risky effects, in order to determine the various risk areas for any daily tobacco use in Europe. At the same time, the present study aims at monitoring any possible spatial differences between these countries, which in general belong to a similar socioeconomic group, and suggest additional control measures.

\section{Materials and Methods}

\subsection{Study Design and Sample}

Data were selected by the World Health Organization (WHO) database for all European countries for 2009 (when the last global monitoring of data took place) (http://www.who.int/gho/en/) [15]. Standardization to age and gender was performed for all countries while seven countries with missing data on some variables were excluded from the analysis (Sweden, Lithuania, Montenegro, Macedonia, Moldova, Azerbaijan and Svalbard -a northernmost part of Norway-). The final study sample consisted from 45 European countries.

Twelve variables were used for the analysis, which were categorized according to the WHO categories [15]: 1) the daily smoking of any tobacco product, which refers to the prevalence (percent \%) estimates for daily smoking of any tobacco product, age and gender-standardized rates for adults aged $>15$ years; 2 ) daily smokers of cigarettes, which refers to the prevalence estimated for daily smoking of cigarettes, age and gender-standardized for adults aged $>15$ years; 3 ) exposure to smoke at home, which is the number of youth exposed to smoke at home per 100 of the youth population (aged 13 - 15). Exposure at home is measured over the last seven days prior to the survey and is defined as people smoking at least once in the presence of the respondent for both genders; 4) annual budget for tobacco control in US\$ at official. This is the amount that was allocated and spent in one budget year, not including what was contained in the budget in US\$, factoring in official exchange rates; 5) number of places smoke-free, which refers to all types of public places based on the national legislations of each country; 6) the existence of national tobacco control programmes or not; 7) offer help to quit tobacco use, which is the implementation status of the Tobacco Dependence Treatment measure, that was classified by grouping countries into five groups. The groupings for this indicator are: Firstly the data that are not reported, no help, NRT and/or some cessation services (neither cost-covered), NRT and/or some cessation services (at least one of which is cost-covered) and finally the existence of National quit line, and both NRT as well as some cessation services cost-covered ; 8) warn about dangers, which is grouped in five categories: no reported data; no warning or warning covering $<30 \%$ of the tobacco package surface; warning covering $\geq 30 \%$ of the tobacco package but no pictures or pictograms, and/or other appropriate characteristics; warning covering $31 \%-49 \%$ of the tobacco package, including pictures or pictograms and other appropriate characteristics; and warning covering $\geq 50 \%$ of the tobacco package, including pictures or pictograms and appropriate characteristics ; 9) anti-tobacco mass media campaigns, which are also grouped in five categories: no reported data; no warning or frequency of $<30 \%$, warning or frequency of $\geq 30 \%$ but poor graphics; warning or frequency of $31 \%-49 \%$ including medium graphics; warning or frequency of $\geq 50 \%$, including appropriate graphics; 10 ) enforce bans on tobacco advertising: no reported data, complete absence of ban, or ban that does not cover national television (TV), radio and print media, ban on national TV, radio and print media only, ban on national $\mathrm{TV}$, radio and print media as well as on some but not all other forms of direct and/or indirect advertising, ban on all forms of direct and indirect advertising; 11) raise taxes on tobacco: Taxes assessed include excise tax, value added tax (VAT), import duty (when the cigarettes were imported) and any other taxes levied. The groupings for this indicator are the following: not reported data, $\leq 25 \%$ of retail price is tax, $26-50 \%$ of retail price is tax, $51-75 \%$ of retail price is tax, $>75 \%$ of retail price is tax; 12) number of health warnings on the packaging that should comply with the legislation around the cigarette packaging.

\subsection{Geo-Epidemiological Analysis}

Geo-data of European countries coastlines and spatial borders were used at the Mercator World projection system, for the whole process of the analysis in (GIS) Arc map 10. The distribution map of daily smoking of any tobacco product was created, demonstrating the variation in the tobacco use across the European countries.

A spatial statistical test for spatial autocorrelation, the Global Moran's Index with filter of Inverse distance and Euclidean distance, was applied on each variable respectively. This was utilised in order to estimate the level of statistical significance (at a 5\% significance level) for each factor, to define their level of autocorrelation by testing if their distribution is random or clustered, and finally to determine which variables will be used in the final regression model [16-19]. Negative values of Moran's I indicated negative spatial autocorrelation and positive values indicate positive autocorrelation, while values range from -1 (indicating perfect dispersion) to +1 (perfect correlation). Zero values 
indicate a random spatial pattern [16, 19].

Furthermore, a spatial regression model was built in order to detect the high risk areas, using the statistically significant variables which had been identified by the Moran's I [16-19]. An Ordinary Least Squares (OLS) regression analysis was run to evaluate the relationships between the several factors and the number of any tobacco use, detecting the high risk areas. It provideda global model of the variables while it creates a single regression equation to represent that process $[18,19]$. Statistical significance is estimated at 0.05 level and Exposure B (Exp B), Std. Deviation, Multiple R-Squared and Adjusted R-Squared (measures of model performance) are also calculated. Exp B refers to the probability of daily tobacco use in relation to the independent factors. These values could be equal to $1,>1$ that means risk, or $<1$ which indicates no risk. Countries were characterized as of no, low, high or very high risk based on the value of the Exp B values; countries with values $<1$ were of no risk, and countries with values $>1$ were characterized proportionally to the degree of risk (as the values were increasing).

\section{Results}

The distribution of daily smoking of any tobacco product is presented in Figure 1. It varied from 16 to $49 \%$ among the European countries, in 2009. Roughly15.38\% of these countries presented with $30-49 \%$ smoking population at a daily base, while the rest $84.62 \%$ of these countries presented with rates lower than $30 \%$. Central - South and Eastern European countries have higher prevalence rates. For instance, Greece and Austria have the highest rates (38-49\%), Bulgaria, Albania, Bosnia Herzegovina, and Russia follow with $30-37 \%$ (light and dark red color). In contrary to that, Norway, Finland, UK, Italy, Portugal, Switzerland are some of the countries that presented with rates less than $23 \%$ (green color). Furthermore, rates of $24-29 \%$ smoking of any tobacco product appeared mainly among countries of central Europe, such as Germany, Poland, France, Belarus, Turkey and Spain (yellow color).

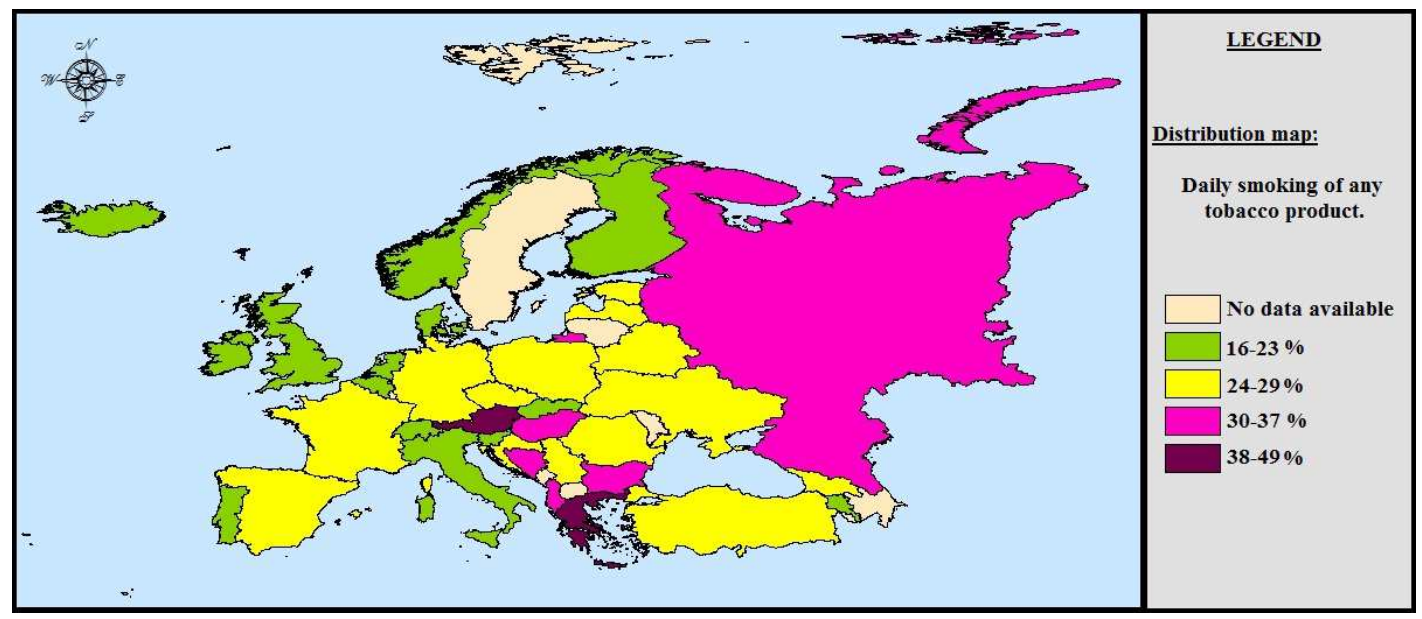

Figure 1. Map of daily smoking of any tobacco product (\%), for all European countries in 2009.

A multi-dimensional analysis is required for the evaluation of a health problem, a disease burden or a public health issue. Consequently, apart from the distribution of the daily smoking of any tobacco product, and its variation, it is important to test other factors which may affect this. At first, these factors were tested through spatial autocorrelation in order to estimate their statistical significance and degree of autocorrelation. Three out of the twelve factors were found to be random. Consequently, nine factors were identified as of high positive autocorrelation and their distribution was clustered (not random). These factors were the following: daily smoking of any tobacco product (Moran's $\mathrm{I}=0.13$; $\mathrm{p}_{\text {value }}<0.001$ ), daily smokers of cigarettes (Moran's $\mathrm{I}=0.10$; $\mathrm{p}_{\text {value }}<0.001$ ), exposure to smoke at home (Moran's $\mathrm{I}=0.5$; $\mathrm{p}_{\text {value }}=0.02$ ), annual budget for tobacco control in US\$ (Moran's $I=0.4 ; p_{\text {value }}=0.04$ ), national tobacco control programmes (Moran's $I=0.9 ; p_{\text {value }}=0.06$ ), offer of help to quit tobacco use (Moran's $\mathrm{I}=0.10 ; \mathrm{p}_{\text {value }}=0.03$ ), anti-tobacco mass media campaigns (Moran's $\mathrm{I}=0.7 ; \mathrm{p}_{\text {value }}<0.03$ ), enforcement of bans on tobacco advertising (Moran's $I=0.08 ; p_{\text {value }}=0.02$ ) and raise of tobacco taxes (Moran's I $=0.12 ; \mathrm{p}_{\text {value }}<0.001$ ). These factors have proved to be of high geo-statistical importance and of clustered pattern, and were used in the next step of the analysis as risk factors for the daily smoking of any tobacco product.

The high risk areas for daily smoking of any tobacco product were identified based not only on the present rates but also on their trends (figure 2). The present model cocalculated the above statistically significant risk factors' presence, for the identification of the high risk areas. Countries were finally grouped into 5 categories. The first group consisted of countries with no data, the second group consisted of countries with no high risk $(\operatorname{Exp} B=0.76 ; 95 \%$ $\mathrm{CI}=0.258-0.936)$, the third group consisted of countries with low risk (Exp $\mathrm{B}=1.20 ; 95 \% \mathrm{CI}=1.032-1.492)$, the forth group consisted of countries with high risk $(\operatorname{Exp} B=2.7 ; 95 \%$ $\mathrm{CI}=1.937-3.013$ ), and the fifth group consisted of countries with very high risk $(\operatorname{Exp} B=2.9 ; 95 \% \mathrm{CI}=2.183-3.326)$. Greece was found to be the only country of very high risk, 
whereas Finland, Norway and the UK proved to be countries with no risk. At the same time, Russia, Albania, Bulgaria, Serbia, Bosnia Herzegovina, Hungary, Turkey and other countries (figure 2, red color) were identified as areas with high risk, with 2.7 times higher risk for daily smoking of any tobacco product. Finally, most of the European countries (mostly of central and western Europe) were found to be of low risk (figure 2, yellow color).

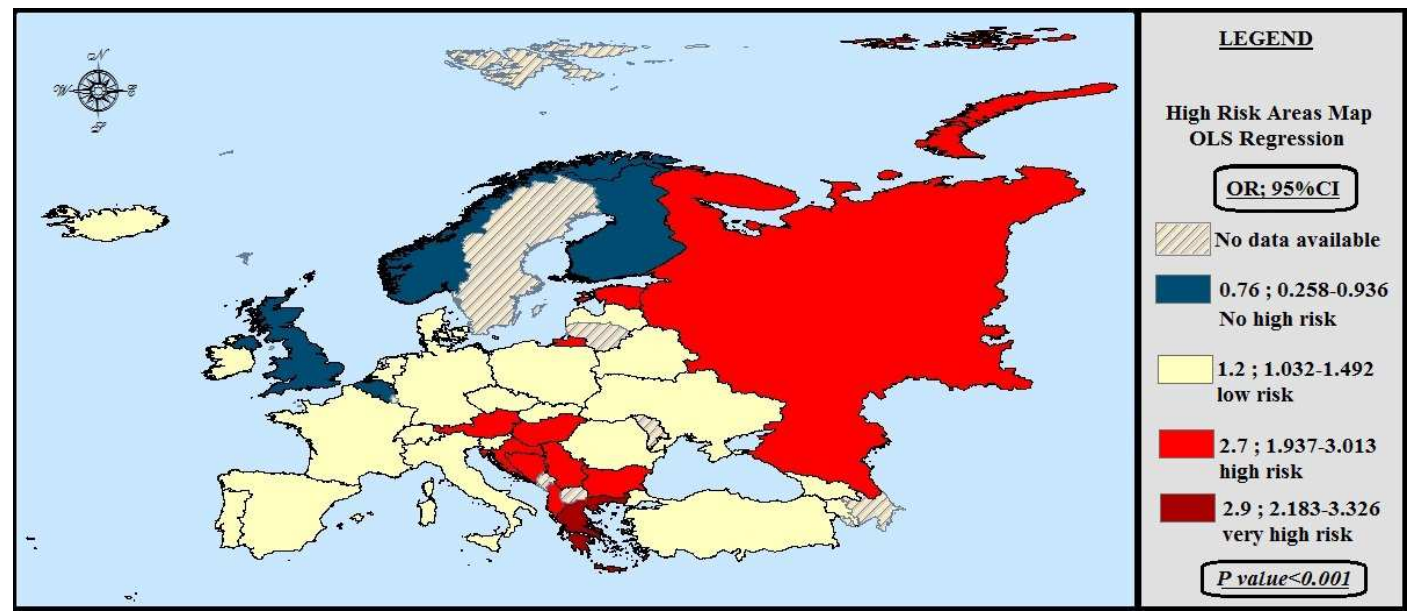

Figure 2. Map of spatial regression (OLS) for the detection of high risk areas, in all European countries in 2009.

Table 1. Spatial autocorrelation Moran's I results for the detection of spatial variance and clustering.

\begin{tabular}{llll}
\hline Variable & Moran's I (Std.Dev) & P value & Random/Clustered \\
\hline 1.Daily smoking of any tobacco product & $0.13(3.74)$ & $<0.001$ & Clustered \\
2.Daily smokers of cigarettes & $0.10(5.22)$ & $<0.001$ & Clustered \\
3.Exposure to smoke at home & $0.5(3.21)$ & 0.02 & Clustered \\
4.Annual budget for tobacco control in US\$ at official & $0.4(2.73)$ & 0.04 & Clustered \\
5.Number of places smoke-free & $-0.22(-0.21)$ & 0.32 & Random \\
6.National tobacco control programmes & $0.9(8.38)$ & 0.06 & Clustered \\
7.Offer help to quit tobacco use & $0.10(5.29)$ & 0.03 & Clustered \\
8. Warn about the dangers of tobacco & $-0.20(-0.19)$ & 0.42 & Random \\
9.Anti-tobacco mass media campaigns & $0.7(4.48)$ & 0.03 & Clustered \\
10.Enforce bans on tobacco advertising & $0.08(7.45)$ & 0.02 & Clustered \\
11.Raise taxes on tobacco & $0.12(6.49)$ & $<0.001$ & Clustered \\
12.Number of health warnings on the packaging & $-0.52(-0.51)$ & 0.04 & Random \\
\hline
\end{tabular}

\section{Discussion}

\subsection{Main Findings}

High spatial variance and heterogeneity are the main characteristics of daily smoking of any tobacco product among the European countries. This fact lead us to test the possible factors which could render a country as a high risk area that requirs direct preventive measures and tobacco control policies. These areas were detected and grouped into five categories according the degree of risk. Most of the under-study factors were found to have a distribution which was clustered (and not random), which indicates a specific spatial pattern which should be explained. The co-existence of these twelve factors was analyzed for the first time in the literature, with the use of a spatial regression model. Furthermore, countries of high risk were mostly countries of high daily smoking of any tobacco product or countries with medium or low rates which yet combined most of the other factors, such as Greece and Bulgaria. Specifically, countries of no organized national tobacco control policies, offer little or no help to their citizens for quitting tobacco. They do not follow strict rules for cigarette and tobacco advertisements while they were found to be in risk for increased tobacco use. At the same time, it is surprising that the number of public smoke-free places, the warnings about the dangers associated with tobacco use, and the number of health warnings on the tobacco packaging do not influence the smokers' behavior. What seem to affect the smokers' behavior are the following: the taxes, the tobacco exposure at home, which is the smokers' close environment, and the help they may have in case they decide to quit smoking. The OLS regression that was used for the estimation of countries with risk is a reliable spatial model that tested the co-existence and influence of the above factors, not only testing them one by one in relation to the tobacco use, but also as a multi-dimensional network.

\subsection{Other Findings in Bibliography}

Approximately $70 \%$ of deaths attributed to tobacco use occur in developing countries, such as European countries [4]. Various studies have contributed to the accumulation of knowledge around tobacco use and the wide range of factors that deter people from smoking [20-25]. For instance, D. Rodriguez et al., suggest that tobacco outlets are more 
concentrated in areas where people with higher risk for negative health outcomes reside. They used spatial analysis methods in order to capture the above trend, showing that tobacco use presents as a spatial dynamic nature [23]. Another study, using GIS application for the examination of tobacco advertising and its spatial density was the one by Vardavas et al. In line with the present study, it demonstrated the effectiveness and reliability of GIS monitoring for tobacco control [24]. Similarly to our study, Mohammed et al., suggested that the banning of tobacco marketing, as recommended by the Framework Convention on Tobacco Control, is likely to reduce tobacco use disparities [25]. Various parameters, included in the tobacco marketing banning of the Framework Convention are also tested in our study, arriving at similar results. For instance, the enforcement of bans on tobacco advertising was found to be of high statistical importance and of clustered spatial pattern, presenting a proportional correlation with smoking of any tobacco product.

\subsection{Impact of This Study and Limitations}

This study contributes to three basic fields related to tobacco control. First of all it distributes the prevalence of smoking of any tobacco product spatially among all European countries. Furthermore, it monitors for the first time, the risk of tobacco smoking in relation to twelve risk factors applying, spatial statistics. Finally, it demonstrates the effectiveness of GIS and geo-epidemiology in the tobacco control research area.

On the other hand, it has a few limitations. The main limitation is that it is actually a secondary analysis of databases. This could produce bias in terms of the accuracy of the findings, although the database chosen is rather reliable. Another point that should be mentioned is that is studies the tobacco use at a country level, so it does not allow us to reach region-related conclusions. However, this study gives important information on tobacco use as well as a clear picture of its epidemiology at a European level. Another study that focuses on the identified high risk areas is required in order to explore their particularities.

\section{Conclusions}

Geo-epidemiology of tobacco use leads to the detection of high risk areas and monitors the phenomenon's dynamics. It contributes to the application of effective tobacco control strategies, with the use of new technologies, highlighting the factors that affect it positively or negatively. This study offered an insight about applying measures for tobacco control and the areas in the European region that are presently in need of direct tobacco use control.

\section{References}

[1] Jha P, Chaloupka FJ. Tobacco control in developing countries. Oxford, UK: Oxford University press 2000.
[2] WHO Report on the Global Tobacco Epidemic. The MPOWER package. Geneva: World Health Organization 2008.

[3] Peto R, Lopez AD, Boreham J, Thun M, Health C. Mortality from smoking in developed countries 1950-2000; indirect estimation from National Vital Statistics. Am $J$ of Epidem 1996; 149: 529-530.

[4] Mackay J, Eriksen M. The Tobacco Atlas. Geneva: World Health Organization 2002.

[5] Li X, Li Q, Dong L, Sun B, Chen J, Jiang Y, et al.. Risk factors associated with smoking behaviour in recreational venues: findings from the International Tobacco Control (ITC) China Survey. Tobacco Control 2010; 19: 130-139.

[6] WHO, Tobacco Free Initiative: Ten facts about tobacco and second-hand smoke. Available at: http://www.who.int/feaures/factfiles/tobacco/tobacco_facts/en/ index.html , Accessed in: 01/05/2012.

[7] Huisman M, Kunst AE, Mackenbach J P. Inequalities in the prevalence of smoking in the European Union: comparing education and income. Prev Med 2005; 40:756-764.

[8] Health Law 76017. Legislation Newspaper of the Government of the Hellenic Democracy, 2nd edition, page 1001, August 1, 2002.

[9] Levy D, Gallus S, Blackman K, Carreras G, Vecchia C, Gorini G. Italy SimSmoke: the effect of tobacco control policies on smoking prevalence and smoking attributable deaths in Italy. BMC Public Health 2012; 12:709.

[10] Thomas RF, Mostashari F, Kerker BD, Miller N, Hajat A, Frankel M. Adult Tobacco Use Levels After Intensive Tobacco Control Measures: New York City, 2002-2003. American Journal of Public Health 2005; 95:1016-1023.

[11] Shibuya K, Ciecierski C, Guindon E, Bettcher DW, Evans DB, Murray CJL. WHO Framework Convention on Tobacco Control: development of an evidence based global public health treaty. $B M J 2003 ; 327: 154$.

[12] Niederdeppe J, Farrelly MC, Wenter D. Media advocacy, tobacco control policy change and teen smoking in Florida. Tob Control 2007;16:1 47-52.

[13] Hamilton WL, Turner-Bowker DM, Celebucki CC, Connolly GN. Recall of anti-tobacco ads impact of warnings advertising: Cigarette advertising in magazines: the tobacco industry response to the Master Settlement Agreement and to public pressure. Tob Control 2002; 11:54-58.

[14] Ross H, Stoklosa M, Krasovsky K. Economic and public health impact of 2007-2010 tobacco tax increases in Ukraine. Tob Control 2010; doi:10.1136/tc.2010.040071.

[15] WHO database, World Health Organization, Available at: http://www.who.int/gho/en/, Accessed in: 28/01/2012.

[16] Anselin L. Local indicators of spatial association - LISA. Geographical analysis 1995; 27: 93-115.

[17] Moran PAP. Notes on Continuous Stochastic Phenomena. Biometrika 1950; 37: 17-23.

[18] Basu S, Reinsel GC. Regression models with spatially correlated errors. Journal of the American Statistical Association 1994; 89: 88-99. 
[19] Mitchell A. The ESRI Guide to GIS Analysis, Vol 2. ESRI Press. 2005.

[20] Sethi JM, Rochester CL. Smoking and chronic obstructive pulmonary disease. Clinics in Chest Medicine 2000; 21: 67-86.

[21] The Health Benefits of Smoking Cessation. A Report of the Surgeon General, DHHS Publication, no. (CDC) 90-8416, US Department of Health and Human Services, Public Health Service, Centers for Disease Control, Centers for Chronic Disease Prevention and Health Promotion, Office on Smoking and Health, Rockville, Md, USA, 1990.

[22] Karp I , O’Loughlin J, Hanley J, Tyndal RF, Paradis G. Risk factors for tobacco dependence in adolescent smokers. Tob Control 2006;15:199-204.
[23] Rodriguez D, Carlos HA, Adachi-Mejia AM, Berke EM, Sargent JD. Predictors of tobacco outlet density nationwide: a geographic analysis. Tob Control 2010; 19: 256-258.

[24] Vardavas CI, Connolly GN, Kafatos AG. Geographical information systems as a tool for monitoring tobacco industry advertising. Tob Control 2006;15:199-204.

[25] Siahpush M, Jones PR, Singh GK, Timsina LR, Martin J. The association of tobacco marketing with median income and racial/ethnic characteristics of neighbourhoods in Omaha, Nebraska. Tob Control 2010;19:256-258. 